\title{
Strong coupling constant at NNLO from DIS data
}

\author{
Anatoly Kotikov*i \\ Joint Institute for Nuclear Research, Russia \\ E-mail: kotikov@theor.jinr.ru \\ Vasily Krivokhizhin \\ Joint Institute for Nuclear Research, Russia \\ E-mail: Vasily.Krivokhizin@lhep.jinr.ru
}

\section{Gonzalo Parente}

Universidade de Santiago de Compostela, Spain

E-mail: gonzalo@fpaxp1.usc.es

\section{Binur Shaikhatdenov}

Joint Institute for Nuclear Research, Russia

E-mail: sbgetheor.jinr.ru

We discuss the results of our recent analysis [1] of deep inelastic scattering data on $F_{2}$ structure function in the non-singlet approximation with next-to-next-to-leading-order accuracy. The study of high statistics deep inelastic scattering data provided by BCDMS, SLAC, NMC and BFP collaborations was performed with a special emphasis placed on the higher twist contributions. For the coupling constant the following value $\alpha_{S}\left(M_{Z}^{2}\right)=0.1167 \pm 0.0022$ (total exp. error) was found.

The XIXth International Workshop on High Energy Physics and Quantum Field Theory

8-15 September 2010

Golitsyno, Moscow, Russia

\footnotetext{
* Speaker.

$\dagger$ The work was supported in part by RFBR grant No.10-02-01259-a. The work of GP was supported in part by the grant Ministerio de Ciencia e Inovacion FPA2008-01177.
} 


\section{Introduction}

It is already a common knowledge that the accuracy of data for DIS structure functions (SFs) allows one to study $Q^{2}$-dependence of logarithmic QCD-inspired corrections and those of powerlike (non-perturbative) nature independently (see for instance [2] and references therein). And this aspect is crucial for the analysis to be performed within some well defined scheme.

In this contribution we present the results of our recent analysis [1] of DIS SF $F_{2}\left(x, Q^{2}\right)$ carried out over SLAC, NMC, BCDMS and BFP experimental data [3] at NNLO of massless perturbative QCD. As in our previous papers $[4,5,6]$ the function $F_{2}\left(x, Q^{2}\right)$ is represented as a sum of the leading twist $F_{2}^{p Q C D}\left(x, Q^{2}\right)$ and the twist four terms:

$$
F_{2}\left(x, Q^{2}\right)=F_{2}^{p Q C D}\left(x, Q^{2}\right)\left(1+\frac{\tilde{h}_{4}(x)}{Q^{2}}\right) .
$$

As is known there are at least two ways to perform QCD analysis over DIS data: the first one (see e.g. [7, 8]) deals with Dokshitzer-Gribov-Lipatov-Altarelli-Parisi (DGLAP) integro-differential equations [9] and let the data be examined directly, whereas the second one involves the SF moments and allows performing an analysis in analytic form as opposed to the former option. In this work we take on the way in-between these two latter, i.e. analysis is carried out over the moments of $\mathrm{SF} F_{2}^{k}\left(x, Q^{2}\right)$ defined as follows

$$
M_{n}^{p Q C D / t w i s t 2 / \cdots}\left(Q^{2}\right)=\int_{0}^{1} x^{n-2} F_{2}^{p Q C D / t w i s t 2 / \cdots}\left(x, Q^{2}\right) d x
$$

and then reconstruct SF for each $Q^{2}$ by using the Jacobi polynomial expansion method $[4,10]$. The theoretical input can be found in the papers $[6,11]$.

\section{A fitting procedure}

The fitting procedure largely follows that used in [6]. With the QCD expressions for the Mellin moments $M_{n}^{k}\left(Q^{2}\right)$ analytically calculated according to the formulæ given above the $\operatorname{SF} F_{2}^{k}\left(x, Q^{2}\right)$ is reconstructed by using the Jacobi polynomial expansion method:

$$
F_{2}^{k}\left(x, Q^{2}\right)=x^{a}(1-x)^{b} \sum_{n=0}^{N_{\max }} \Theta_{n}^{a, b}(x) \sum_{j=0}^{n} c_{j}^{(n)}(\alpha, \beta) M_{j+2}^{k}\left(Q^{2}\right),
$$

where $\Theta_{n}^{a, b}$ are the Jacobi polynomials and $a, b$ are their parameters to be fitted. A condition imposed on the latter is the requirement of the error minimization while reconstructing the structure functions.

Since a twist expansion starts to be applicable only above $Q^{2} \sim 1 \mathrm{GeV}^{2}$ the cut $Q^{2} \geq 1 \mathrm{GeV}^{2}$ is imposed on the experimental data throughout. The MINUIT program [12] is used to minimize two variables

$$
\chi_{S F}^{2}=\left|\frac{F_{2}^{e x p}-F_{2}^{\text {teor }}}{\Delta F_{2}^{\text {exp }}}\right|^{2}, \quad \chi_{\text {slope }}^{2}=\left|\frac{D^{\text {exp }}-D^{\text {teor }}}{\Delta D^{\exp }}\right|^{2},
$$

where $D=d \ln F_{2} / d \ln \ln Q^{2}$. The quality of the fits is characterized by $\chi^{2} / \mathrm{DOF}$ for the SF $F_{2}$. Analysis is also performed for the slope $D$ that serves the purpose of checking the properties of fits.

We use free normalizations of the data for different experiments. For a reference set, the most stable deuterium BCDMS data at the value of the beam initial energy $E_{0}=200 \mathrm{GeV}$ is used. 


\begin{tabular}{|l|c|c|c|c|c|}
\hline$Q_{\min }^{2}$ & $\begin{array}{c}N \text { of } \\
\text { points }\end{array}$ & HTC & $\chi^{2}\left(F_{2}\right) / \mathrm{DOF}$ & $\alpha_{s}\left(90 \mathrm{GeV}^{2}\right) \pm$ stat & $\alpha_{s}\left(M_{Z}^{2}\right)$ \\
\hline \hline 1.0 & 797 & No & 2.20 & $0.1767 \pm 0.0008$ & 0.1164 \\
2.0 & 772 & No & 1.14 & $0.1760 \pm 0.0007$ & 0.1162 \\
3.0 & 745 & No & 0.97 & $0.1788 \pm 0.0008$ & 0.1173 \\
4.0 & 723 & No & 0.92 & $0.1789 \pm 0.0009$ & 0.1174 \\
5.0 & 703 & No & 0.92 & $0.1793 \pm 0.0010$ & 0.1176 \\
6.0 & 677 & No & 0.92 & $0.1793 \pm 0.0012$ & 0.1176 \\
7.0 & 650 & No & 0.92 & $0.1782 \pm 0.0015$ & 0.1171 \\
8.0 & 632 & No & 0.93 & $0.1773 \pm 0.0018$ & 0.1167 \\
9.0 & 613 & No & 0.93 & $0.1764 \pm 0.0022$ & 0.1163 \\
\hline \hline 1.0 & 797 & Yes & 0.98 & $0.1772 \pm 0.0027$ & 0.1167 \\
\hline
\end{tabular}

Table 1: $\alpha_{s}\left(M_{Z}^{2}\right)$ and $\chi^{2}$ in the case of the combined analysis (HTCs stands for higher twist corrections).

\section{Results}

Since the gluon distribution function is not taken into account in the nonsinglet approximation, the analysis is substantially easier to conduct; hence the cut on the Bjorken variable $(x \geq 0.25)$ imposed where gluon density is believed to be negligible. The starting point of the evolution is taken to be $Q_{0}^{2}=90 \mathrm{GeV}^{2}$. These $Q_{0}^{2}$ values are close to the average values of $Q^{2}$ spanning the corresponding data. The previous experience tells us that the maximal value of the number of moments to be accounted for is $N_{\max }=8$ [4] (though we check $N_{\max }$ dependence just like in the NLO analysis) and the cut $0.25 \leq x \leq 0.8$ is imposed everywhere.

In $[6,1]$ the cuts on the kinematic variable $Y=\left(E_{0}-E\right) / E_{0}$ have been imposed so as to exclude BCDMS data with large systematic errors. Here $E_{0}$ and $E$ are lepton initial and final energies, respectively. Upon excluding the set of data with large systematic errors considerably higher values of $\alpha_{s}\left(M_{Z}^{2}\right)$ are obtained and rather mild dependence of its values on the choice of $Y$ cut is observed. For more details we refer to $[1,6]$. Once these cuts are applied, a full set of data consists of 797 points.

To verify a range of applicability of perturbative QCD we start with analyzing the data without a contribution of twist-four terms (which means $F_{2}=F_{2}^{p Q C D}$ ) and perform several fits with the cut $Q^{2} \geq Q_{\min }^{2}$ gradually increased. From Table 1 it is seen that unlike the NLO analysis the quality of the fits starts to appear fairly good from $Q^{2}=3 \mathrm{GeV}^{2}$ onwards (at NLO, it starts at $Q^{2}=10$ $\left.\mathrm{GeV}^{2}[6]\right)$. Then, the twist-four corrections are added and the data with the usual cut $Q^{2} \geq 1 \mathrm{GeV}^{2}$ imposed upon is fitted. It is clearly seen that as in the NLO case (see [6]) here the higher twists do sizably improve the quality of the fit, with insignificant discrepancy in the values of the coupling constant to be quoted below.

The parameter values of the twist-four term are presented in Table 2. Note that these for $\mathrm{H}_{2}$ and $D_{2}$ targets are obtained in separate fits by analyzing SLAC, NMC and BCDMS datasets taken together. For illustrative purposes we visualize those for the hydrogen data in Fig. 1, where the 


\begin{tabular}{|l||c|c|}
\hline$x$ & $\tilde{h}_{4}(x)$ for $H_{2} \pm$ stat & $\tilde{h}_{4}(x)$ for $D_{2} \pm$ stat \\
\hline \hline 0.275 & $-0.183 \pm 0.020$ & $-0.197 \pm 0.009$ \\
0.35 & $-0.149 \pm 0.028$ & $-0.171 \pm 0.015$ \\
0.45 & $-0.182 \pm 0.029$ & $-0.033 \pm 0.031$ \\
0.55 & $-0.236 \pm 0.052$ & $0.142 \pm 0.057$ \\
0.65 & $-0.180 \pm 0.135$ & $0.295 \pm 0.108$ \\
0.75 & $-0.177 \pm 0.182$ & $0.303 \pm 0.158$ \\
\hline
\end{tabular}

Table 2: HTC parameter values obtained in NNLO analysis.

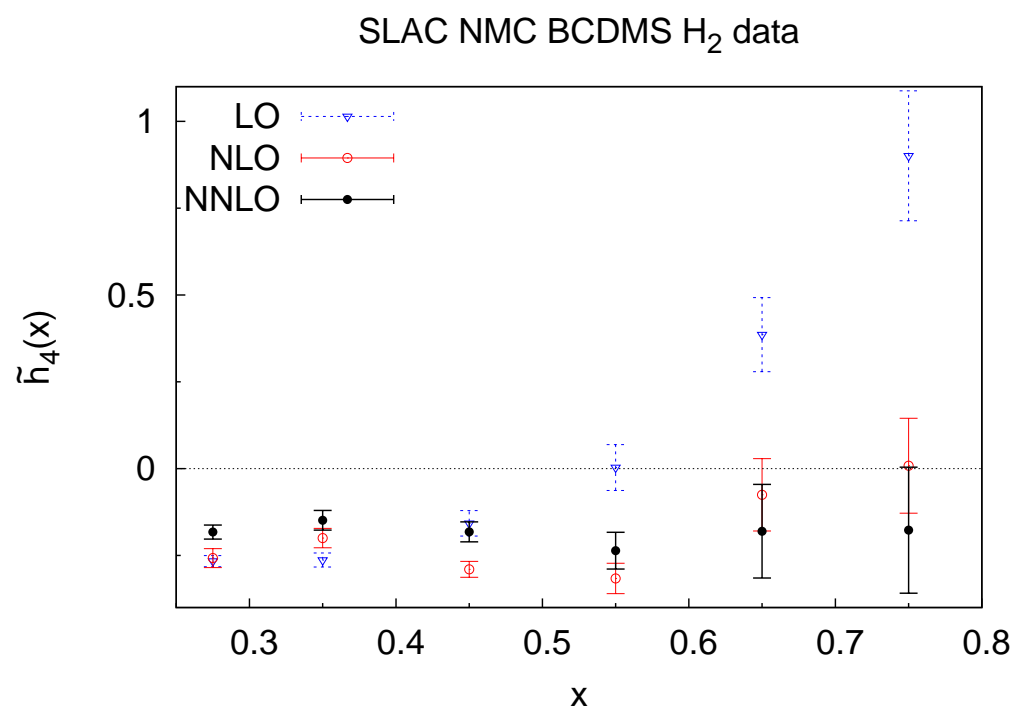

Figure 1: Comparison of the HTC parameter $\tilde{h}_{4}(x)$ obtained at LO, NLO and NNLO for hydrogen data (the bars indicate statistical errors).

HTCs obtained at NLO and NNLO levels are seen to be compatible with each other within errors.

We would like to note that the cut of the BCDMS data, which has increased the $\alpha_{s}$ values (see Fig. 1 in [1]) improves considerably agreement between perturbative QCD and experimental data. Indeed, the HTCs, that are nothing else but the difference between the twist-two approximation (i.e. pure perturbative QCD contribution) and the experimental data, are seen to become considerably smaller at NLO and NNLO levels as compared with both the NLO higher twist terms obtained in [7] and and the results of analysis obtained with no $Y$-cuts imposed on the BCDMS data (see Fig. 2).

\section{Conclusions}

In the paper [1] the Jacobi polynomial expansion method developed in $[4,10]$ was used to perform analysis of $Q^{2}$-evolution of DIS structure function $F_{2}$ by fitting all the existing to date reliable fixed-target experimental data that satisfy the cut $x \geq 0.25$. Based on the results of fitting, the QCD 


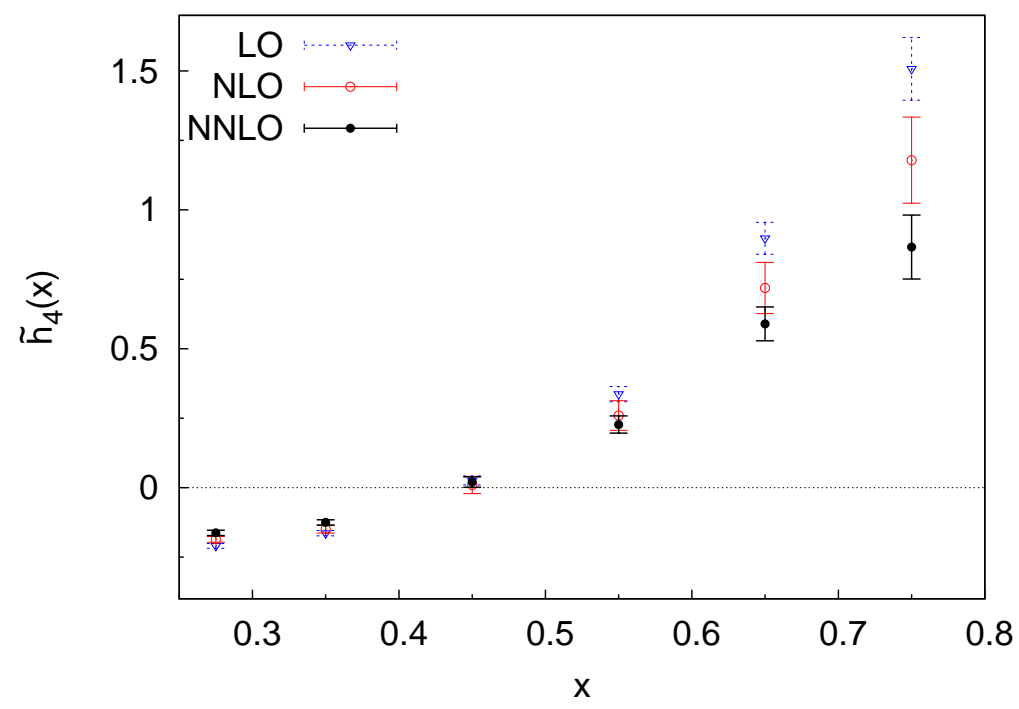

Figure 2: Comparison of the HTC parameter $\tilde{h}_{4}(x)$ obtained at LO, NLO and NNLO for hydrogen data when no $Y$ cuts imposed on the BCDMS data.

coupling constant value at the normalization point was evaluated. Starting with the reanalysis of BCDMS data by cutting off the points with large systematic errors it was shown $[1,6]$ that the values of $\alpha_{s}\left(M_{Z}^{2}\right)$ rise sharply with the cuts on systematics imposed. The values of $\alpha_{s}\left(M_{Z}^{2}\right)$ obtained in various fits are in agreement with each other. An outcome is that quite a similar result for $\alpha_{s}\left(M_{Z}^{2}\right)$ was obtained [1] in the analysis performed over BCDMS (with the cuts on systematics) and the rest of the data, thus permitting us to fit available data altogether.

It turns out that for $Q^{2} \geq 3 \mathrm{GeV}^{2}$ the formulae of pure perturbative QCD (i.e. twist-two approximation accompanied by the target mass corrections) are enough to achieve good agreement with all the data analyzed. The reference result is then found to be

$$
\begin{aligned}
\alpha_{S}\left(M_{Z}^{2}\right) & =0.1167 \pm 0.0008 \text { (stat) } \pm 0.0018 \text { (syst) } \pm 0.0007 \text { (norm) } \\
& =0.1167 \pm 0.0021 \text { (total exp. error) }
\end{aligned}
$$

Upon adding twist-four corrections, QCD (i.e. first two coefficients of Wilson expansion) and the data are shown to be consistent with each other already at $Q^{2}=1 \mathrm{GeV}^{2}$, where the Wilson expansion begins to be applicable. This way we obtain for the coupling constant at $Z$ mass peak:

$$
\begin{aligned}
\alpha_{s}\left(M_{Z}^{2}\right) & =0.1167 \pm 0.0007 \text { (stat) } \pm 0.0020 \text { (syst) } \pm 0.0005 \text { (norm) } \\
& =0.1167 \pm 0.0022 \text { (total exp. error) }
\end{aligned}
$$

Note that the above values (4.1) and (4.2) are to some extent stable [13] under the application of the "frozen" [14] and analytic [15] modifications of the strong coupling constant, which as a rule lead to similar results (see [16]).

Note also that our results (4.1) and (4.2) for $\alpha_{s}\left(M_{Z}^{2}\right)$ are in good agreement with the world 
average value for the coupling constant presented in the review [17], ${ }^{1}$

$$
\alpha_{s}\left(M_{Z}^{2}\right)=0.1184 \pm 0.0007
$$

Concerning the contributions of higher twist corrections in the present work the well-known $x$-shape of the twist-four corrections while going from intermediate to large values of the Bjorken variable $x$ is well reproduced.

A.K. is indebted to organizers for the possibility to present the talk which this paper is based on.

\section{References}

[1] B.G. Shaikhatdenov, A.V. Kotikov, V.G. Krivokhizhin, and G. Parente, Phys. Rev. D81 (2010) 034008 .

[2] M. Beneke, Phys. Rept. 317 (1999) 1.

[3] SLAC Collab., L.W. Whitlow et al., Phys. Lett. B282 (1992) 475; NM Collab., M. Arneodo et al., Nucl. Phys. B483 (1997) 3. BCDMS Collab., A.C. Benevenuti et al., Phys. Lett. B223 (1989) 485; Phys. Lett. B237 (1990) 592; Phys. Lett. B195 (1987) 91. BFP Collab.: P.D. Mayers et al., Phys. Rev. D34 (1986) 1265.

[4] V.G. Krivokhizhin et al. Z. Phys. C36 (1987) 51; Z. Phys. C48 (1990) 347.

[5] A.V. Kotikov, G. Parente and J. Sanchez Guillen, Z. Phys. C58 (1993) 465; G. Parente, A.V. Kotikov and V.G. Krivokhizhin, Phys. Lett. B333 (1994) 190; A.L. Kataev et al., Phys. Lett. B388 (1996) 179; Phys. Lett. B417 (1998) 374;

[6] V.G. Krivokhizhin, A.V. Kotikov, Yad.Fiz. 68 (2005) 1935; Phys.Part.Nucl. 40 (2009) 1059.

[7] M. Virchaux and A. Milsztajn, Phys. Lett. B274 (1992) 221.

[8] STEQ Collab., W.K. Tung et al., JHEP 0702 (2007) 053; A.D. Martin et al., Phys. Lett. B652 (2007) 292; M. Gluck, C. Pisano and E. Reya, Phys. Rev. D77 (2008) 074002; S. Alekhin et al., Phys. Rev. D 81 (2010) 014032 [arXiv:0908.2766 [hep-ph]].

[9] V.N. Gribov and L.N. Lipatov, Sov. J. Nucl. Phys. 15 (1972) 438; L.N. Lipatov, Sov. J. Nucl. Phys. 20 (1975) 94; G. Altarelli and G. Parisi, Nucl. Phys. B126 (1977) 298; Yu.L. Dokshitzer, JETP 46 (1977) 641.

[10] G. Parisi and N. Sourlas, Nucl. Phys. B151 (1979) 421; I.S. Barker et al., Nucl. Phys. B186 (1981) 61; Z. Phys. C19 (1983) 147; I.S. Barker and B.R. Martin, Z. Phys. C24 (1984) 255.

[11] A.V. Kotikov, Phys.Part.Nucl. 38 (2007) 1. [Erratum-ibid. 38 (2007) 828].

[12] F. James and M. Ross, "MINUIT", CERN Computer Center Library, D 505, Geneve, 1987.

[13] G. Cvetic, A. Y. Illarionov, B. A. Kniehl and A. V. Kotikov, Phys. Lett.B679 (2009) 350; A.V. Kotikov V.G. Krivokhizhin, and B.G. Shaikhatdenov, arXiv:1008.0545 [hep-ph].

[14] B. Badelek, J. Kwiecinski, and A. Stasto, Z. Phys. C74 (1997) 297.

[15] D.V. Shirkov and I.L. Solovtsov, Phys. Rev. Lett. 79 (1997) 1209.

[16] A.V. Kotikov, A.V. Lipatov, and N.P. Zotov, J. Exp. Theor. Phys. 101 (2005) 811.

[17] S. Bethke, Eur. Phys. J. Phys. C64 (2009) 689.

\footnotetext{
${ }^{1}$ It should be mentioned that this analysis was carried out over the data coming from the various experiments and in different orders of perturbation theory, i.e. from NLO up to $\mathrm{N}^{3} \mathrm{LO}$.
} 